\title{
Optical Fiber Vertical Seismic Profile using DAS (Distributed Acoustic Sensing) Technology
}

\author{
Tsunehisa KIMURA ${ }^{1}$, Gareth LEES $^{1}$ and Arthur HARTOG ${ }^{1}$ \\ ${ }^{1}$ Schlumberger Fiber Optics Technology Center, Southampton, UK
}

\begin{abstract}
Distributed optical fiber sensing technologies have been evolved over more than 30 years starting with DTS (Distributed Temperature Sensing). DTS is now commonly used for well monitoring in the oil and gas business. The subsequently developed DAS (Distributed Acoustic Sensing) technology was introduced more than 5 years ago to meet the demands of pipeline monitoring and intrusion detection. The latest optical fiber sensing technology now allows DAS to record borehole seismic signals including VSPs (Vertical Seismic Profiles). This system is called hDVS (heterodyne Distributed Vibration Sensing) to distinguish it from the pipeline monitoring system. Unlike conventional VSP recording tools, which usually use electromagnetic accelerometers or geophones, hDVS/DAS uses optical fiber as a vibration sensor. Because optical fiber can be deployed along the entire well depth as a sensor, either permanently (e.g., using control line) or temporarily (hybrid logging cable), the acquisition time required for hDVS VSP can be as short as a few minutes, which is essentially the time required for firing of the seismic source, compared with several hours to days for conventional VSPs, including the time required for multiple tool settings. The core part of the optical fiber is made of high-silica glass, so the high-temperature version of optical fiber is widely available rated to more than $200^{\circ} \mathrm{C}$ and even above $500{ }^{\circ} \mathrm{C}$ in specialized cases. Hence, an optical fiber sensor can be deployed on a permanent basis in high-temperature environments, where conventional geophones cannot be used. This is an absolutely new way of acquiring borehole seismic data by using fiber-optic technology.
\end{abstract}

\section{INTRODUCTION}

Multilevel borehole seismic imager tools including VSI (Versatile Seismic Imager) have been commonly used to save time in recording VSPs for more than 15 years. However, because the sensing is limited to the location of the geophone, the borehole seismic tool must be moved from the bottom section of the well up to the surface in stages (tool settings) to record VSP data for the entire well (Fig. 1). A massive number of seismic source shots may be required, in proportion to the number of tool settings. The rig-up and rig-down time for multilevel borehole seismic tools, which can have 20 or more sensors, increases significantly as the number of sensors deployed into the well increases. Hence, the ability to reduce acquisition time using conventional methods is limited.

An additional limitation, due to the nature of electronics system, is that the maximum temperature at which conventional borehole seismic tools can be deployed without using Dewar flasks is $200{ }^{\circ} \mathrm{C}$ or even lower.

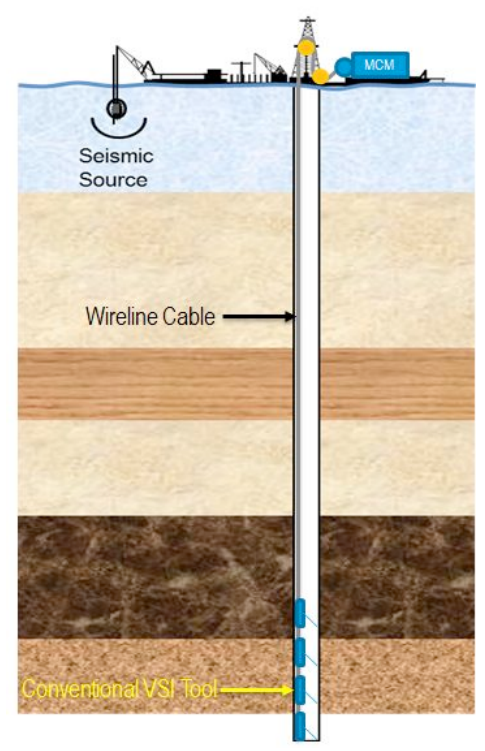

Figure 1 Schematic of conventional VSP.

The hDVS/DAS application uses optical fiber as a vibration sensor, which can be deployed along the entire well depth as a sensor, either permanently or temporarily (Fig. 2). The acquisition time required for hDVS VSP can be as short as a few minutes, 
which is essentially the time required for firing the seismic source 20 to 50 times.

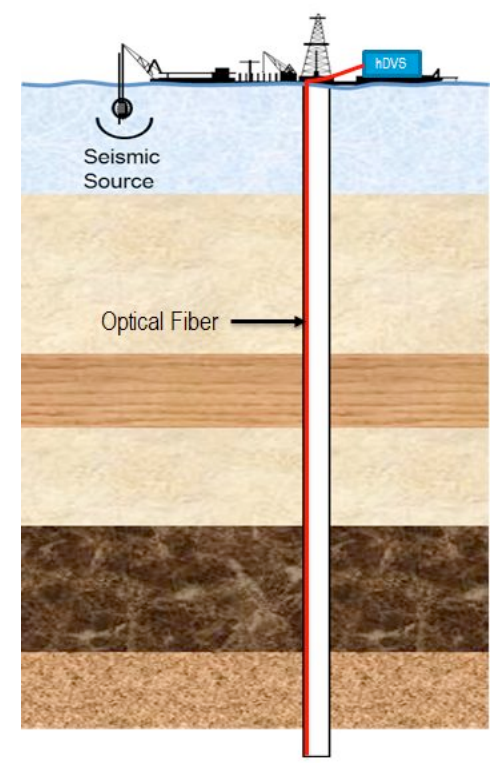

Figure 2 Schematic of optical fiber VSP.

The availability of high-temperature versions of optical fiber, rated above $200{ }^{\circ} \mathrm{C}$, enables recording VSPs in geothermal wells or very deep hydrocarbon wells, where conventional geophones cannot be used. The optical fibers can be daisy-chained from one well to the other until significant optical loss is observed, so that a multiple-well VSP can be recorded using a single seismic source in a few minutes, whereas this acquisition would take several days using conventional methods and also have a high associated cost.

\section{PRINCIPLE}

OTDR (Optical Time-Domain Reflectometry) forms the basis for the hDVS system. The basic principle is that a short pulse of light travels down an optical fiber and, as the pulse of light propagates, a small amount of energy scatters at each elemental section of the fiber. A small fraction (about $0.25 \%$ for the fibers typically used for hDVS) of the scattered light is recaptured by the waveguide and guided back to the launching end. The returning scattered light is acquired as a function of the time elapsed from launching the probe pulse. Because the group velocity of light in the fiber is known, the timing of a particular sample of the acquired trace can be related to the location in the fiber at which the light was scattered.

In practice, we use an arrangement that compares the phase of backscattered light from closely spaced fiber regions. This results in a stronger signal and a more linear mapping of sensor output to local strain than is possible using the backscattered power only.
The arrangement is illustrated in Fig. 3.

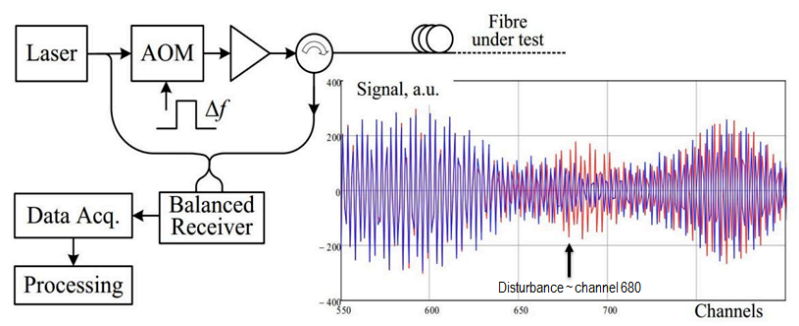

Figure 3 Arrangement of the hDVS system and (inset) two successive coherent OTDR traces ${ }^{1)}$.

The output of a highly coherent laser is split into two paths. In the upper path, the light is modulated by an AOM (Acousto-Optic Modulator) that defines a sequence of probe pulses from the incoming light. The AOM also shifts the frequency of light by $\Delta f$, and the probe pulses are amplified in an erbium-doped-fiber optical amplifier. The circulator passes the pulses from the amplifier to the sensing fiber but directs the backscatter to a third port, where it is mixed with a sample of the laser light at a 50/50 coupler (arriving through the lower optical path from the laser); the two outputs of the coupler are connected to a balanced receiver. The signal at the balanced receiver is at the beat frequency $\Delta f$, the difference between the frequency of the laser and the backscattered light. The mixing (or heterodyne) process preserves the phase of the backscattered light, which is, however, shifted down to a convenient frequency in the radio-frequency range. Thus, the backscatter has a pseudo-periodic appearance at $\Delta f$, with the information carried in its amplitude and phase. The inset in Fig. 3 shows an example of two backscattered traces in the form of the digitized signal from the balanced receiver. As can be seen, this takes the form of a quasi-periodic signal. The successive traces overlap up to approximately channel 680, where a disturbance has occurred resulting in a phase change for all subsequent channels.

We process the signal in software after digitizing the output of the receiver. Specifically, the phase is estimated at each location along the fiber and differentiated across an interval to provide a measure of the change in length of that interval. The differentiated phase at a given location, tracked over a sequence of probe pulses, provides a measure of the dynamic strain at that location. Typically, the pulse duration is 65 ns (i.e., the probe pulse occupies about $6.5 \mathrm{~m}$ of fiber), and the differentiation interval corresponds to about $10 \mathrm{~m}$ of fiber. The differentiation interval defines the spatial resolution of the system and, in our approach, 
can be selected at the time of processing the data ${ }^{2}$.

\section{SYTEM OVERVIEW}

\section{(1) hDVS system}

The hDVS system consists of six major components as shown in Fig. 4.

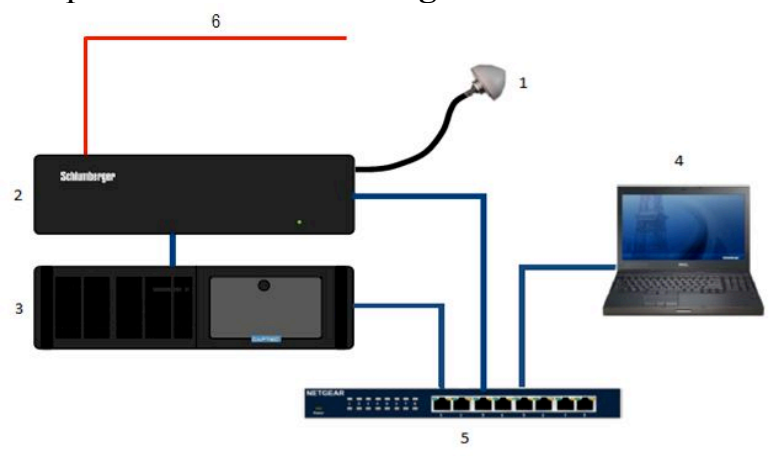

Figure 4 hDVS system schematic.

1. GPS antenna-used for receiving satellite signals to provide an accurate time stamp

2. hDVS optical unit-used for acquiring hDVS data

3. hDVS processing server unit - used for sending commands to the hDVS optical unit and for processing and storing data

4. Computer - used to communicate to the hDVS processing server unit to start/stop acquisition, play back the data, and edit parameters. A seismic source controller is also connected to the computer, allowing "Fire Order" and "Time Break" communication.

5. LAN Switch-used to enable all the instruments to communicate with one another

6. Optical fiber-vibration measurement sensor

\section{(2) Current hDVS specifications}

The specification of the current hDVS system as of early 2016 is shown on Table 1 .

Table 1 hDVS specifications

\begin{tabular}{|l|l|}
\hline \multicolumn{1}{|c|}{ Parameter } & \multicolumn{1}{c|}{ Value } \\
\hline Native measurement type & Strain \\
\hline Operating principle & Coherent phase measurement \\
\hline Operating wavelength & $1550 \mathrm{~nm}$ \\
\hline Range & $10 \mathrm{~km}$ (typical) \\
\hline Typical noise floor & $0.1 \mathrm{~nm} / \mathrm{m}$ (in laboratory) \\
\hline Spatial sampling & $2 \mathrm{~m}, 5 \mathrm{~m}, 10 \mathrm{~m}$ \\
\hline Gauge length & $10 \mathrm{~m}, 20 \mathrm{~m}, 30 \mathrm{~m}, 40 \mathrm{~m}$ \\
\hline Sampling time & $0.1 \mathrm{~ms}, 0.25 \mathrm{~ms}, 0.5 \mathrm{~ms}, 1 \mathrm{~ms}$, \\
& $2 \mathrm{~ms}$ \\
\hline Low-frequency limit & $5 \mathrm{~Hz}($ depends on application) \\
\hline Fiber type & $\mathrm{SMF}$ (preferred), MMF \\
\hline Supply voltage & $100-240 \mathrm{~V}, 50-60 \mathrm{~Hz}$ \\
\hline Operating temperature & $0-45^{\circ} \mathrm{C}$ \\
\hline
\end{tabular}

\section{FUNCTIONAL CHECK}

To check the functionality of the system, we always conduct an operational check of the system by attaching an optical fiber cable prior to recording seismic data. Fig. 5 shows an example in which hDVS data was recorded for $30 \mathrm{sec}$ over the optical fiber total length of $5,700 \mathrm{~m}$. A strain signal was applied to the bottom $400 \mathrm{~m}$ section of the fiber. A tapping signal was observed for $7 \mathrm{sec}$, starting from $11 \mathrm{sec}$ after the start of recording.

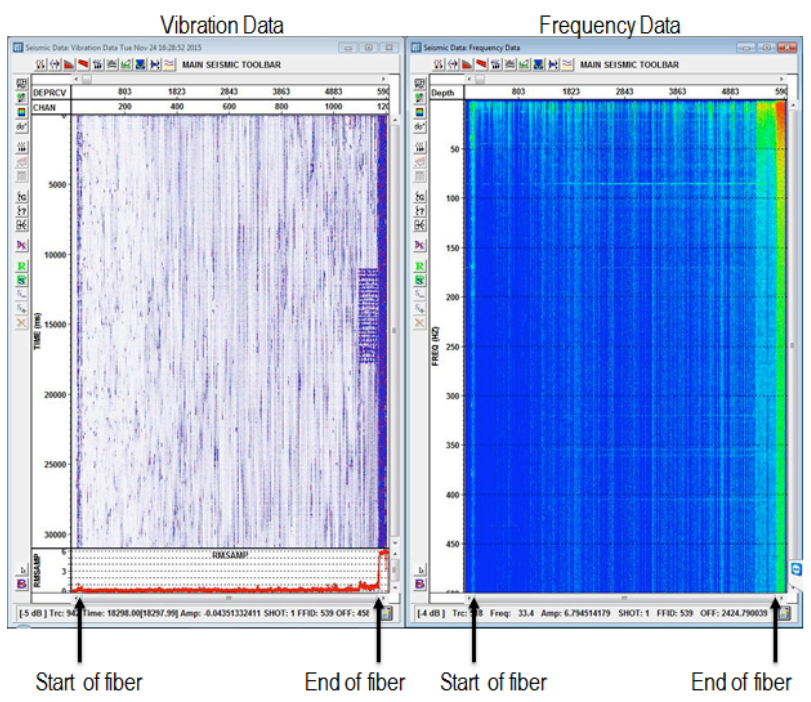

Figure 5 hDVS operational check data.

\section{FIELD TRIAL-PERMANENT FIBER}

\section{(1) Test setup}

SMF (Single-Mode Fiber) and MMF (Multi-Mode Fiber) are permanently installed (cemented) in four vertical wells at the Ketzin pilot site owned by GFZ (Geo Forschungs Zentrum, in German) as shown in Fig. 6. Each well has a depth of 700-800 m.

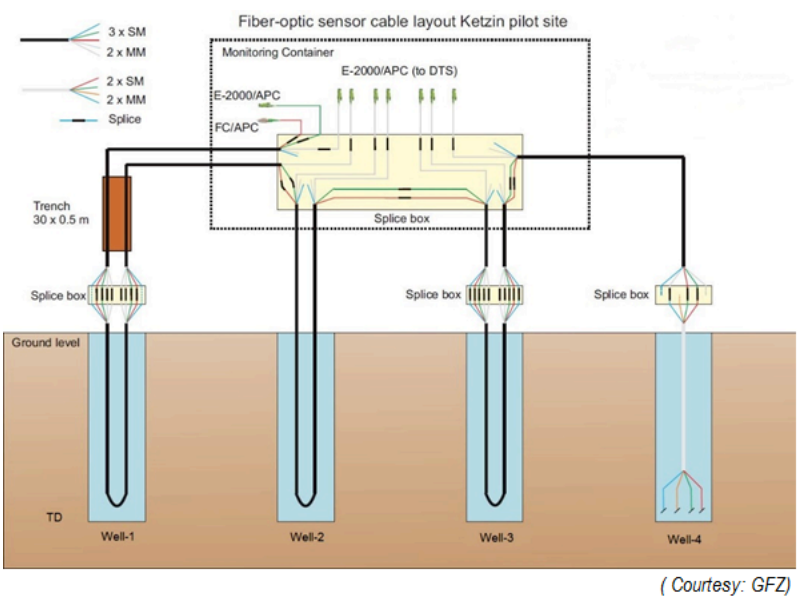

Figure 6 Permanent fiber installation at 
Ketzin pilot site ${ }^{3)}$.

The SMF are daisy-chained to each well for a total length greater than $5 \mathrm{~km}$ whereas each MMF is installed individually.

A single AWD (Accelerated Weight Drop) source, which is not a standard seismic source for the oil and gas industry, was used for this test because the wells are shallow (Fig. 7). To improve the S/N (Signal-to-Noise Ratio), 50 shots were recorded at each source position.

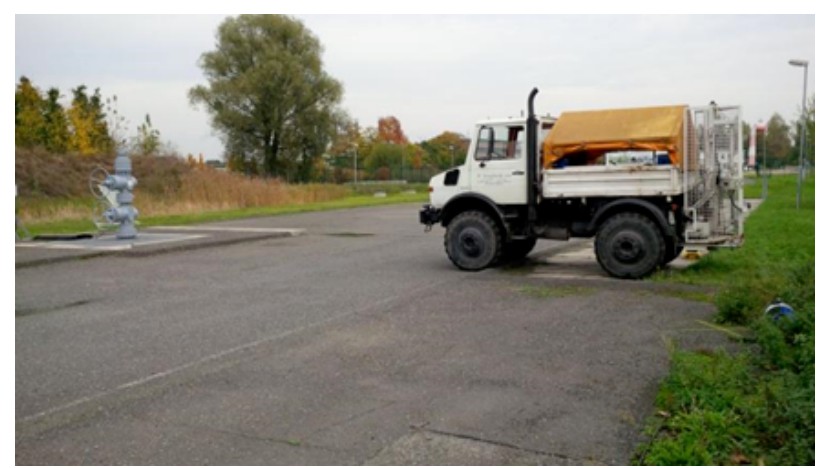

Figure 7 Single AWD source located at zero-offset position and the Well-1 wellhead.

\section{(2) Raw data}

Raw data acquired through the daisy-chained SMF is shown in Fig. 8. The $\mathrm{X}$-axis is the fiber length and the Y-axis is time. Well-1, Well-2 and Well-3 have doubled SMF with a loop-back termination, so that mirror images are shown, whereas Well-4 has single-ended SMF. Because the AWD source was located nearest to the Well-1 wellhead, high ground roll was observed there. The cement quality at shallow sections was marginal, so some ringing is shown due to poor fiber cable coupling to the formation. It is known that Well-4 has the best cement quality, and indeed the data recorded over Well-4 has the highest $\mathrm{S} / \mathrm{N}$ and less significant ringing effects. Hence, the data recorded in Well-4 was processed further.

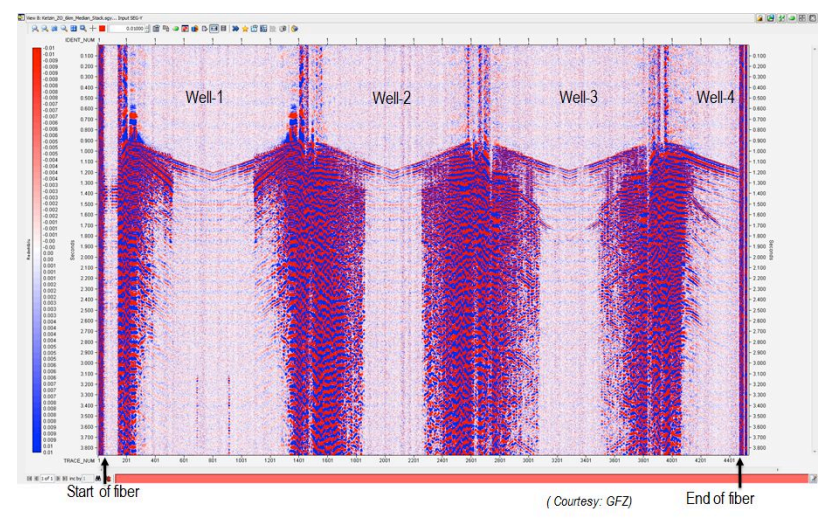

Figure 8 hDVS raw data through daisy-chained

\section{(3) Processed data}

SMF (50-shot linear stack).

First, the hDVS data recorded through the SMF and MMF within Well-4 were compared. SMF is preferable for hDVS measurement because it does not show the speckle effects that can be present in $\mathrm{MMF}^{4)}$. However, MMF can be used as long as the optical signal loss is acceptable and nonlinear effects are not shown. Fig. 9 shows a comparison of SMF and MMF data acquired in Well-4.

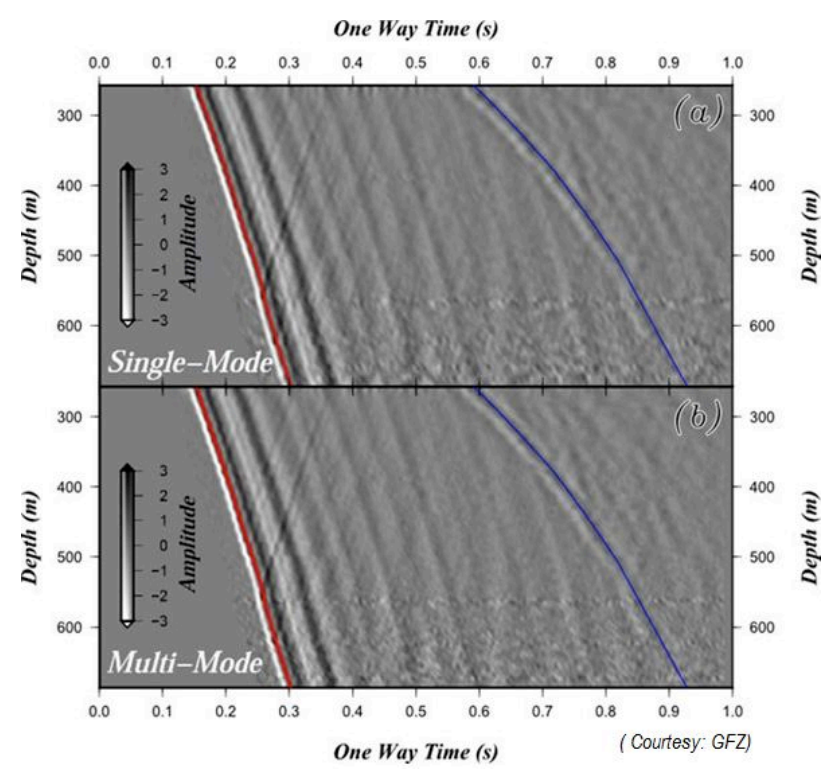

Figure 9 Comparison SMF and MMF (50-shot median stack).

These two sets of data are similar and have equivalent data quality. The portion of the daisy-chained SMF in Well-4 was the far-end section of the SMF, which has total length of more than $5 \mathrm{~km}$. On the other hand, the MMF in Well-4 is less than $1 \mathrm{~km}$ in length.

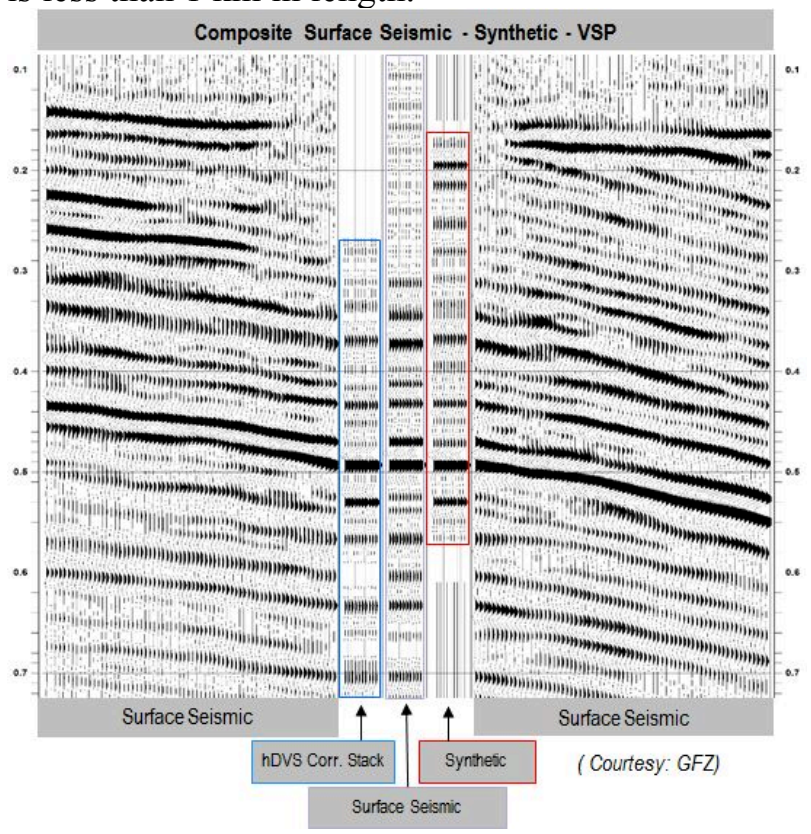


Figure 10 VSP corridor stack.

Fig. 10 is a composite of the surface seismic, hDVS corridor stack, and a synthetic seismogram derived from sonic data. There was no conventional VSP data available for Well-4 to compare. It is clearly seen that the hDVS corridor stack and synthetic are well matched and able to eliminate multiples in the surface seismic data.

\section{FIELD TRIAL-HYBRID CABLE}

\section{(1) Test setup}

Hybrid heptacable is a cable providing seven electrical connections for conventional wireline logging as well as optical fiber connections ${ }^{5)}$. Fig. 11 shows an example of hybrid heptacable, for which two of the conductors contain SMF inside. The electrical properties and the temperature ratings are similar to conventional modern heptacable. However, the cable's safe working load is reduced to protect the optical fibers from being damaged under high tension.
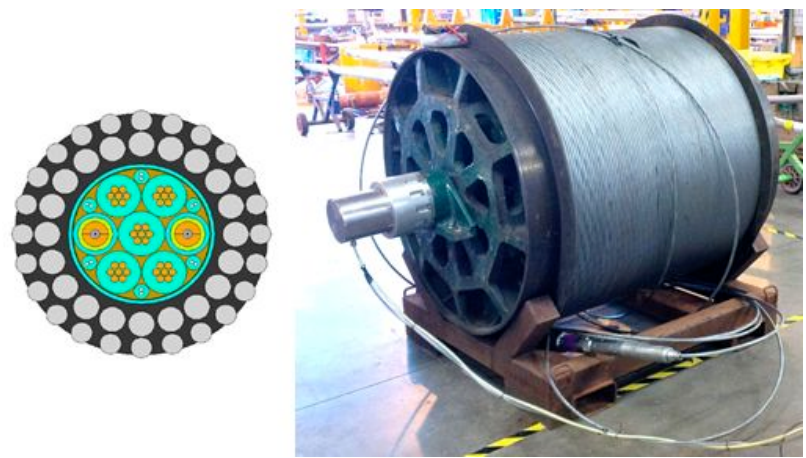

Figure 11 Hybrid heptacable.

Unlike cemented permanent optical fiber, hybrid heptacable stays in a wellbore for a short period of time during logging operations, so that the coupling of the cable to the wellbore is highly dependent on the deviation of the well, the tension on the cable and the properties of the well fluid. In other words, cable coupling is limited in the case of a vertical cased hole well filled with water.

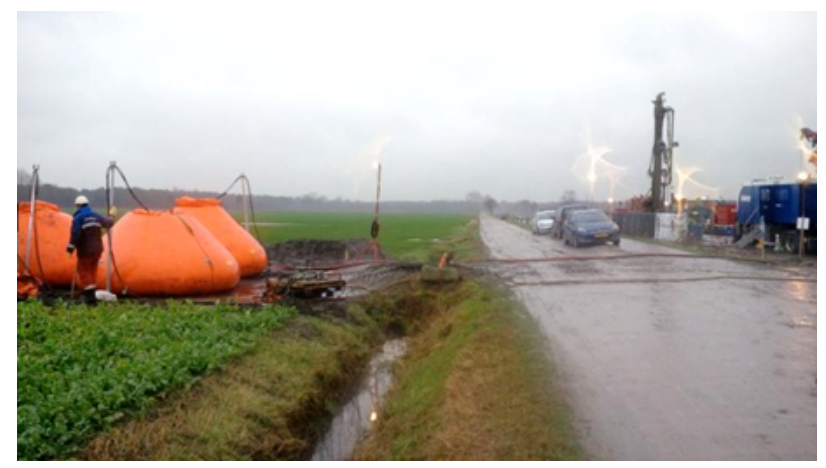

Figure 12 Airguns PAT source located at zero-offset position.

The well depth of the openhole well owned by NIRAS (Nationale Instelling voor Radioactief Afval en verrijkte Splijtstoffen, in Dutch) is relatively shallow $(\sim 600 \mathrm{~m})$, so a source consisting of thee airguns in a PAT (Portable Airgun Tank) ${ }^{6}$ was used with a TRISOR gun controller as shown in Fig. 12. Each airgun has a chamber volume of 54 in $^{3}$ and the air pressure used was 40 bar.

\section{(2) Raw data}

The hDVS data was acquired while two shuttles VSI were connected to the hybrid heptacable at the bottom of the openhole well. The VSI required only five shots of source firing at each tool station; however, it required 40 stations to cover the entire well. It took about 3.5 hours for VSI acquisition.

On the other hand, hDVS required 20 shots to improve the $\mathrm{S} / \mathrm{N}$ by using stack processing, but only one cable position was sufficient to cover the entire well. It took only $3.5 \mathrm{~min}$ for hDVS acquisition. The raw data is shown in Fig. 13, with $\mathrm{X}$-axis depth and the $\mathrm{Y}$-axis time.

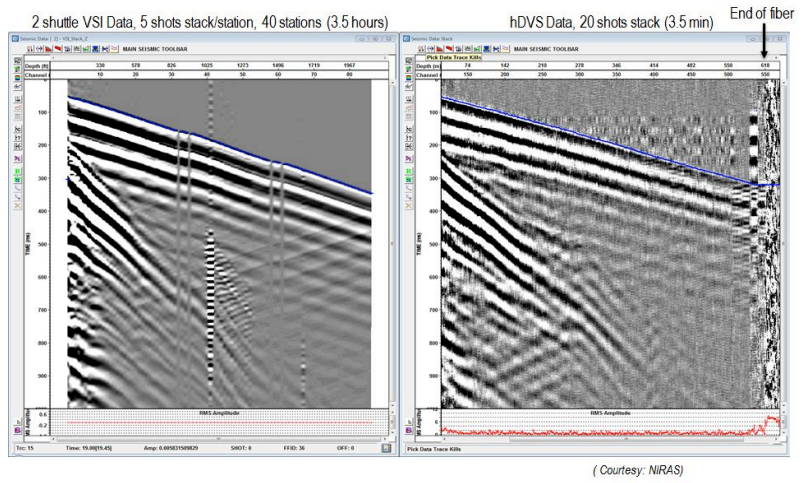

Figure 13 Raw data VSI and hDVS.

The blue lines in Fig. 13 indicate the first break of the P-wave. VSI data on the left shows a high $\mathrm{S} / \mathrm{N}$ however, there are several invalid data areas (ringing) due to hole rugosity or partial cement behind the casing. The ringing in the middle of the image occurred right below casing shoe.

hDVS data on the right has a lower $\mathrm{S} / \mathrm{N}$ than VSI data, but the first break is consistent, and the major characteristic of the seismic image is similar to that of the VSI data.

\section{(3) Processed Data}

Comparison of the transit time vs. depth plot between VSI and hDVS is shown in Fig. 14. The hDVS line closely overlays the VSI line apart from the near-surface and near-bottom sections, which were affected by the surface noise, cable coupling or insufficient energy of the seismic source used. 
About $75 \%$ of the data points differ by less than 1 $\mathrm{ms}$ in the computed arrival time. From our experience, using a higher-energy seismic source such as vibroseis, would increase the transit time vs. depth plot agreement to $90 \%$.

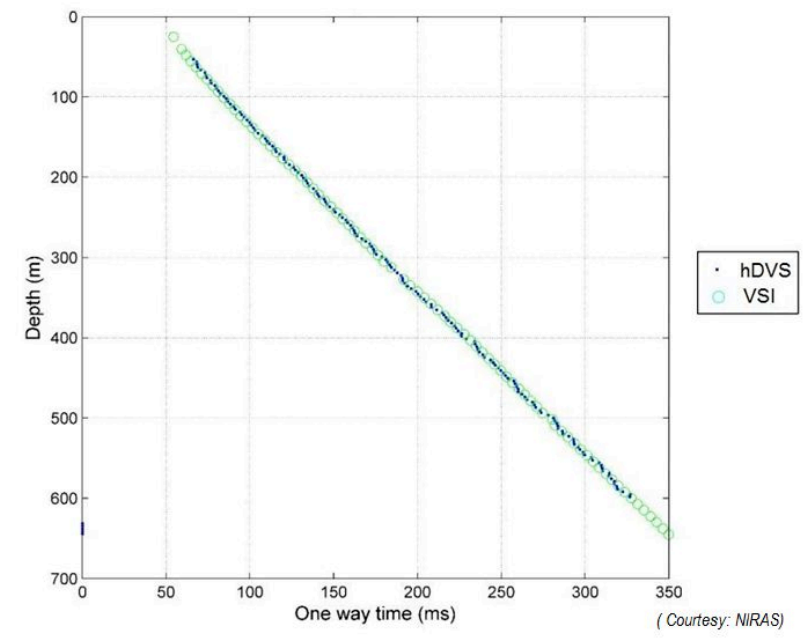

Figure 14 Transit time vs. depth plot.

The comparison of processed seismic images between VSI and hDVS is shown in Fig. 15. Indeed, VSI image has superior data quality. However, the hDVS image shows reasonable data quality and matches the major characteristics of the VSI imager such as downgoing and upgoing P-waves and downgoing S-wave.

The major, advantageous difference is that hDVS required only $1 / 60$ of VSI recording time and 1/10 of VSI airguns shots to record this useful hDVS image.

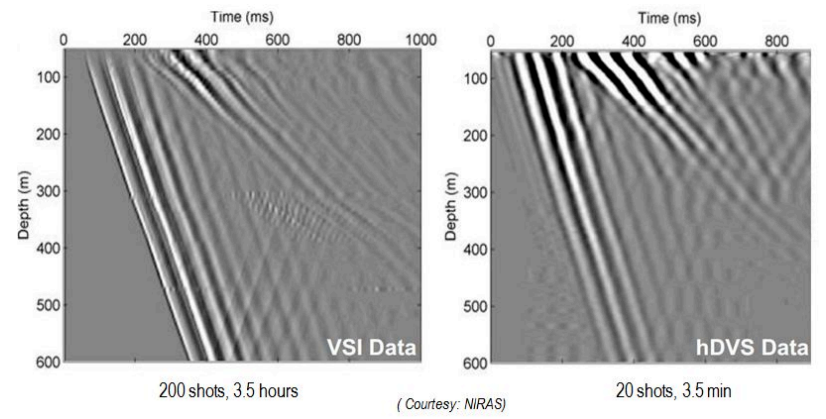

Figure 15 Processed data for the VSI and. hDVS.

\section{CONCLUSIONS}

Zero-offset VSP data recorded with the hDVS system using permanently installed (cemented) optical fiber and hDVS system is of equivalent data quality to that of a conventional VSP tool. If the fiber length is short, (e.g., $1000 \mathrm{~m}$ ), hDVS data recorded through MMF is equivalent to that of SMF. In this scenario, VSP data can be recorded at any time without rig as long as a seismic source is available, which enables low-cost time-lapse VSP operations.

Zero-offset VSP data was also recorded using the hDVS system in combination with hybrid heptacable and provided equivalent data quality to that of a conventional VSP tool if sufficient cable coupling is achieved and sufficient seismic source effort used. hDVS can record zero-offset VSP data in the order of minutes, whereas conventional methods require in the order of hours; hence, hDVS can significantly reduce rig time and cost for drilling wells.

Using high-temperature optical fiber, hDVS is able to record seismic events within very hot environments such as $500{ }^{\circ} \mathrm{C}$ or even higher, where conventional methods cannot be used.

ACKNOWLEDGMENT: We thank GFZ and NIRAS for permission to show recorded the hDVS data.

\section{REFERENCES}

1) A.H. Hartog, O.I. Kotov, L.B. Liokumovich, The Optics of Distributed Vibration Sensing, 2nd EAGE Workshop on Permanent Reservoir Monitoring, July 2-5, 2013

2) B.G. Frignet, A.H. Hartog, Optical Vertical Seismic Profile on Wireline Cable, SPWLA 55th Annual Logging Symposium, May 18-22, 2014

3) Götz, J., Lüth, S., Henninges, J. and Reinsch, T. (2015) Using a Fibre Optic Cable as Distributed Acoustic Sensor for Vertical Seismic Profiling at the Ketzin $\mathrm{CO}_{2}$ Storage Site. 77th EAGE Conference and Exhibition 2015, 01 June 2015, DOI: $10.3997 / 2214-4609.201413363$

4) D. Davies, A.H. Hartog and K. Kader, "Distributed vibration sensing system using multimode fibre", US 7,668,411 B2, 2010.

5) J. Varkey, R. Mydur, N. Sait et al., "Optical fiber cables for wellbore applications", US7324730 B2. 2008.

6) M. Clark, D. Mackie, and B. Frignet, "Portable Airgun Tank-A new approach to Land Borehole Seismic using airguns", 2nd EAGE Borehole Geophysics Workshop, Malta, 2013. 